\title{
Automated visual inspection algorithm for the reflection detection and removing in image sequences
}

\author{
Sizyakin Roman ${ }^{\mathrm{a}, \mathrm{c}}$, Voronin Viacheslav ${ }^{\mathrm{a}}$, Gapon Nikolay ${ }^{\mathrm{a}}$, Aleksandr Zelensky ${ }^{\mathrm{b}}$, and \\ Aleksandra Pižurica ${ }^{\mathrm{c}}$ \\ ${ }^{a}$ Lab. "Mathematical methods of image processing and intelligent computer vision systems" \\ Don State Technical University, Rostov-on-Don, Russia \\ ${ }^{\mathrm{b}}$ Moscow State University of Technology "STANKIN", Moscow, Russia \\ ${ }^{c}$ Department Telecommunications and Information Processing, IPI-TELIN-imec, \\ Ghent University, Ghent, Belgium
}

\begin{abstract}
Specular reflections are undesirable phenomena that can impair overall perception and subsequent image analysis. In this paper, we propose a modern solution to this problem, based on the latest achievements in this field. The proposed method includes three main steps: image enhancement, detection of specular reflections, and reconstruction of damaged areas. To enhance and equalize the brightness characteristics of the image, we use the alpha-rooting method with adaptive choice of the optimal parameter-alpha. To detect specular reflections, we apply morphological filtering in the HSV color space. At the final stage there is a reconstruction of damaged areas using adversarial neural networks. This combination makes it possible to quickly and effectively detect and remove specular reflections, which is confirmed by a series of experiments given by the experimental section of this work.
\end{abstract}

Keywords: Specular reflections, image enhancement, alpha-rooting, HSV color space, morphological filtering, adversarial neural network, image inpainting

\section{INTRODUCTION}

Specular reflections are an integral part of most images, and often serve as an auxiliary tool for its analysis [1]. The types of specular reflections can be divided into two main types: useful and undesirable. The useful types include reflections that help in the analysis of the image. For example, analyzing the shape of objects in a three-dimensional reflection space can help determine the curvature or roughness of a surface. Various types of reflections are also useful when generating or creating images similar to real [2] images. Another type of reflection should include reflections that violate the overall integrity of the picture. This occurs when the brightness of an area in the image exceeds the acceptable level, which causes the true structure of the image to disappear. Such distortions can lead to an increase in the number of false segments in the segmentation problem [3]. The problem of excessive light reflection is also found in the field of endoscopic medical imaging [4]. The formation of unwanted glare in endoscopic analysis is associated with a moistened surface of the internal organs, the properties of which are similar to the mirror surface, which in turn leads to such reflections. Effective detection and removal of unwanted reflections in a short period of time is a difficult task. This is due to the fact that the reflections can have a complex shape, uneven brightness, arbitrary area and coincide/merge with the structure of the original image.

In this paper, we propose a fast and efficient method for detecting and removing specular reflections. The proposed method is a three-stage procedure: (a) image enhancement using an adaptive alpha-rooting algorithm, (b) an efficient reflection detection algorithm in the HSV color space and (c). We use the image enhancement procedure for improving the visual appearance of the image and provide "better" transform for future automated detection and segmentation. The basic idea is to apply the modified $\alpha$-rooting image enhancement approach for different image blocks and a parameter optimization via the measure of enhancement (EME). For automatic specular reflection detection stage, we use space transformation, binarization processing, and morphology filtration. At the final stage, a reconstruction procedure based on the use of an adversarial neural network is applied to all detected areas with undesirable specular reflections. 


\section{RELATED WORK}

Specular reflection detection algorithm can be classified regarding two properties: thresholding and feature-based methods (fig. 1) [5]. The specular detection is the subject in different applications has investigated by many researchers. In some cases, the thresholding algorithms show the effectiveness of detecting specular regions [6,7]. Authors in [8] used the thresholding for the saturation and intensity components that reduce the highlights in laparoscopic surgery. Zimmerman-Moreno et al. proposed to use probabilistic segmentation on hue and saturation components for detection specular regions [9]. The color balance adaptive thresholds and nonlinear filtering allow detecting high intense specular

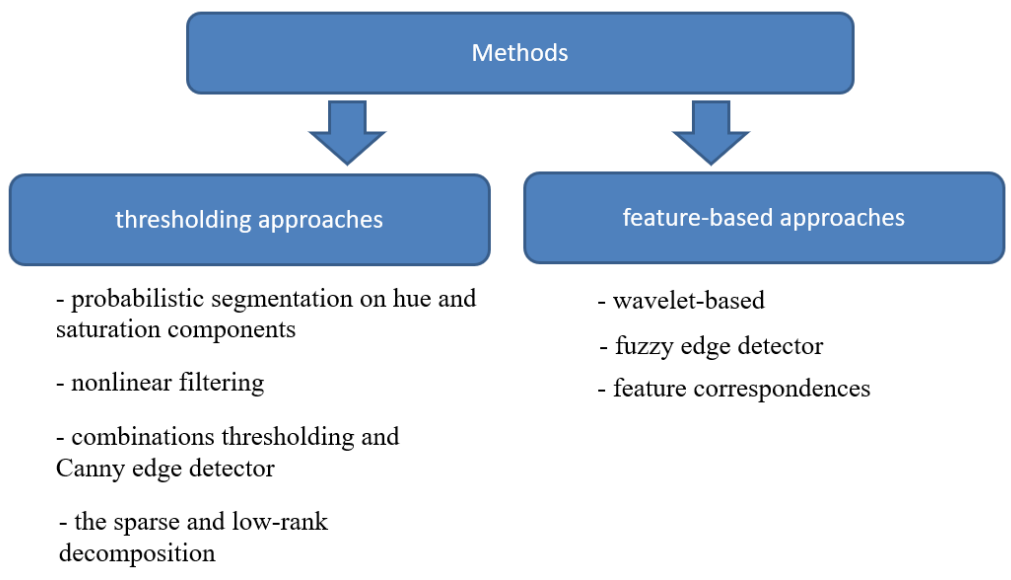

Figure 1. Key specular reflection detection methods classification

highlights in [10]. The authors in [11] proposed combinations thresholding and Canny edge detector for large and small regions respectively. Stehle et al. used the brightest as the global threshold in the YUV color space. A specular highlights algorithm based on nonlinear filtering and color image thresholding proposed in [6]. In works [12] and [13] autors used thresholding, combined with a mask dilation process, to detect specular reflections. In work [14], the authors use two normalized color spaces (HSV and RGB) for which mean and variance are computed for each region around each pixel of the input image.The variance and mean are then used for the support vector machine classification. In the work of [15] authors in the initial phase, used the correction of the histogram to normalize brightness characteristics of the image. After that, the image is rendered to binary form using an adaptive selection threshold that depends on the area of the image.

Each of these methods has strong and weak points. The main disadvantages of the known methods come from the fact that most of them are unable to detect large specular reflection regions and are more suitable for the small high intense specular highlights.

So, the weaknesses of traditional methods are:

- $\quad$ extremely sensitive to parameters.

- $\quad$ fails to detect in case a large range of brightness light sources and complex texture organs surface.

- $\quad$ mostly limited to their chosen field of applications and computational complexity.

- unable to detect large specular reflection regions and are more suitable for the small high intense specular highlights.

The methods for reconstructing damaged/unwanted areas can be conditionally divided into 3 main groups: (a) gradient methods [16], (b) methods based on the search for self-similar areas in the image [17], and (c) methods based on machine learning [18]. The disadvantages of gradient methods include the lack of structuredness/textuality of the restored area. Methods based on the search for self-similar blocks are devoid of this drawback. The main disadvantage of this group of methods is the inability to properly repair damaged areas in the absence of the necessary blocks in the intact image area. Machine learning-based methods currently allow the most efficient reconstruction of damaged areas. The main advantage is the ability to generate new objects for reconstruction, even if they are not on the damaged image. The disadvantages include the training process, which sometimes requires significant time costs, as well as the need to maintain the similarity of the restored image with the images from the training set. 
The objective of our work is to develop a new specular reflection detection algorithm with the subsequent reconstruction of the damaged areas.

\section{PROPOSED METHOD}

To detect the artifact of specular highlights and reflection in images, we developed an automatic solution that we show in Fig. 2. We propose a solution to process images to contrast enhancement based on combined local and global image processing, detect the specular reflection regions using color space transformation and binarization processing, and then reconstruct the damaged areas using adversary neural network.

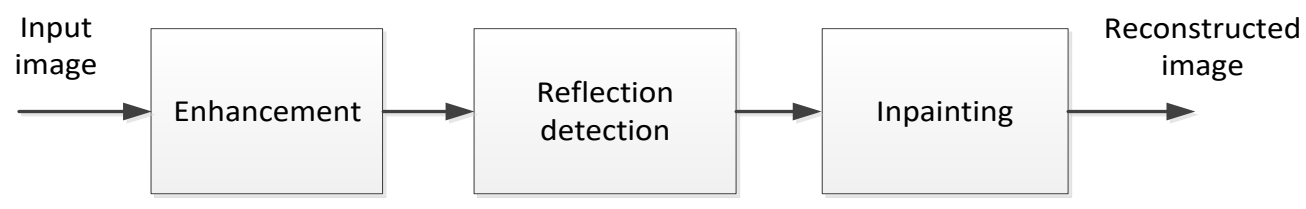

Figure 2. System block diagram

Many images may suffer from the following degradations: poor contrast due to poor illumination or finite sensitivity of the imaging device and electronic sensor noise. Image enhancement is the image processing that the results are more suitable for display or further image analysis [19-22]. The solution of the last task is very important when image enhancement procedure is used as a preprocessing step for other image processing techniques such as detection, recognition, and visualization.

\subsection{Image enhancement}

We use image enhancement algorithm based on combined local and global image processing (See Algorithm 1) [23].

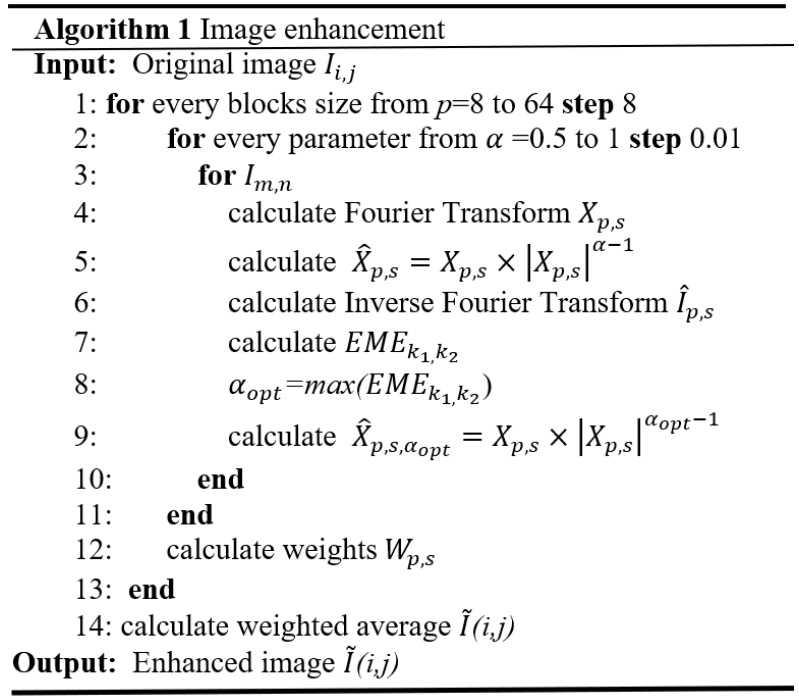

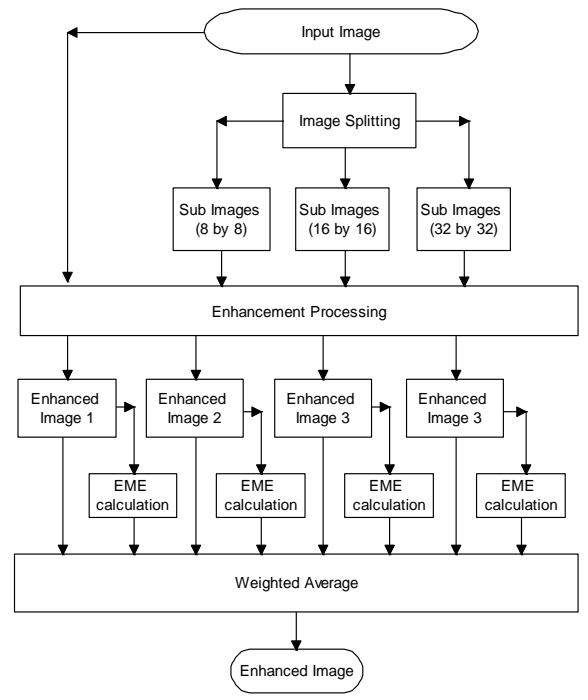

Figure 3. System block diagram

The block diagram of the proposed enhancement algorithm is shown in figure 3 . The basic idea is to apply $\alpha$-rooting image enhancement approach for different image blocks [24, 25]. For every block, we use the transform-based enhancement algorithm base on the $\alpha$-rooting and magnitude reduction method [19]:

$$
\hat{X}(p, s)=X(p, s) \times|X(p, s)|^{\alpha-1}=|X(p, s)|^{\alpha} \times e^{i \theta(p, s)}
$$

where $X(p, s)$ is the transform coefficients of the image, $\alpha$ is a user defined operating parameter, $\theta(p, s)$ is the phase of the transform coefficients. 
The $\alpha$-rooting transform depends on the parameter $\alpha$. We are choosing the best (optimal) enhancement image through optimization of measure enhancement (EME) introduced by Agaian [26]:

$$
E M E_{k_{1}, k_{2}}=\max \left(\frac{1}{k_{1} \times k_{2}} \times \sum_{l=1}^{k_{1}} \sum_{k=1}^{k_{2}} 20 \times \log \frac{x_{\max ; k ; l}^{\omega}}{x_{\min ; k ; l}^{\omega}}\right)
$$

where $x_{\max ; k ; l}^{\omega}$ and $x_{\min ; k ; l}^{\omega}$ respectively are the minimum and maximum of the image $x(m, n)$ inside the block $\omega_{k, l}$.

For every patch of the image, we apply $\alpha$-rooting algorithm with the value of alpha that maximizes the value of EME. Figure 4 demonstrates the image enhancement results obtained by the proposed algorithm.

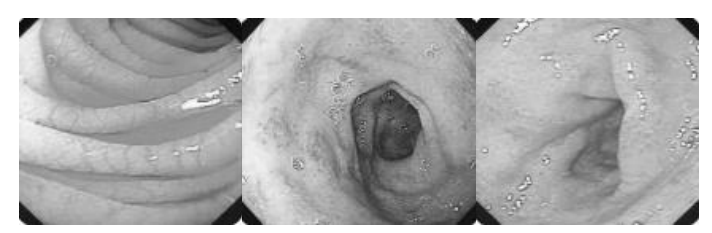

a)

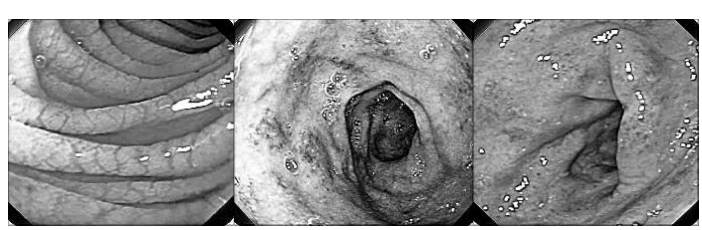

b)

Figure 4. Examples of image enhancement. a) Original images, b) Enhanced images

\subsection{Specular reflection detection}

A map must be generated that contains ones where the image is undisturbed and zeros where specular reflections occur. The proposed specular reflection detection algorithm shows in Fig. 5. The first part of our solution involves transform from RGB to into the HSV color space by applying the linear transform. The specular highlights are very bright, so the affected pixels show high luminance in the HSV color space. We study RGB, YUV, YIQ, HSV color spaces for this task.

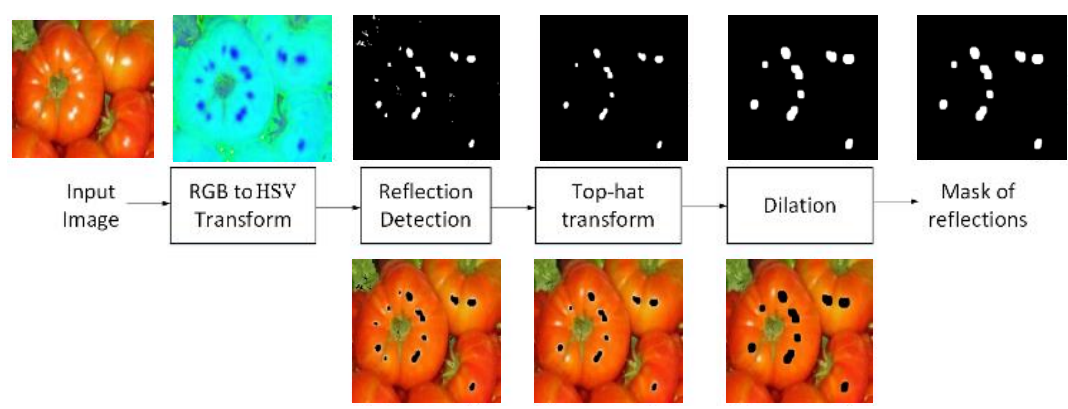

Figure 5. The proposed specular reflection detection algorithm.

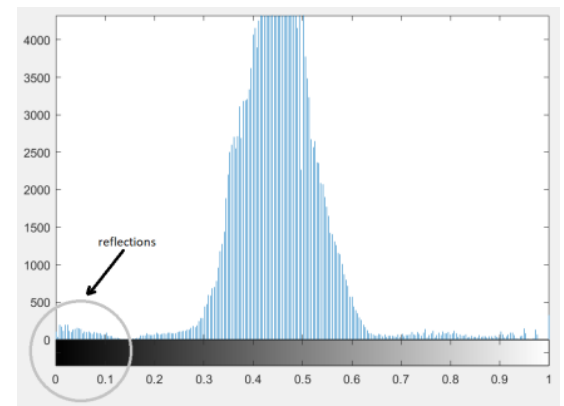

Figure 6. The histogram for S-component.

We chose S-component in HSV model to detect large specular reflection regions and the small high intense specular highlights. This color attribute was used because specular highlights are characterized by the local coincidence of intense brightness and unsaturated color. HSV is alternative representations of the RGB color model with components Hue, Saturation, Value. This color space mixes the different colors, with the saturation dimension resembling various shades of brightly colored paint, and the value dimension resembling the mixture of those paints with varying amounts of black or white paint. The saturation image shows less false detections of the specular highlights pixel then other components.

Then we perform histogram analysis on a single image to identify suitable intensity and saturation thresholds. For this purpose is to apply the Otsu method with a threshold value equal to 10. The histogram for S-component with the threshold is shown in Fig. 6.

Since there are often white rings around the affected pixels in the reconstructed image, we reduce the segmented areas by morphological processing. For this purpose, the top-hat transform is applied. This transforms base on the 
opening operation and returns an image containing those objects that are smaller than the structuring and are brighter than their surroundings. After that, we use the dilation to enlarge detected regions.

\subsection{Image reconstruction}

At the final stage, we use generatively adversarial networks to reconstruct the damaged areas of the image. Adversarial neural networks in the standard case are two neural networks: generator G and discriminator D. Task of the generator $\mathrm{G}$ is to generate an image that discriminator $\mathrm{D}$ cannot distinguish from the real one. The main advantage of the adversarial architecture as opposed to the standard encoder-decoder architecture is the generation of sharp images by the generator.

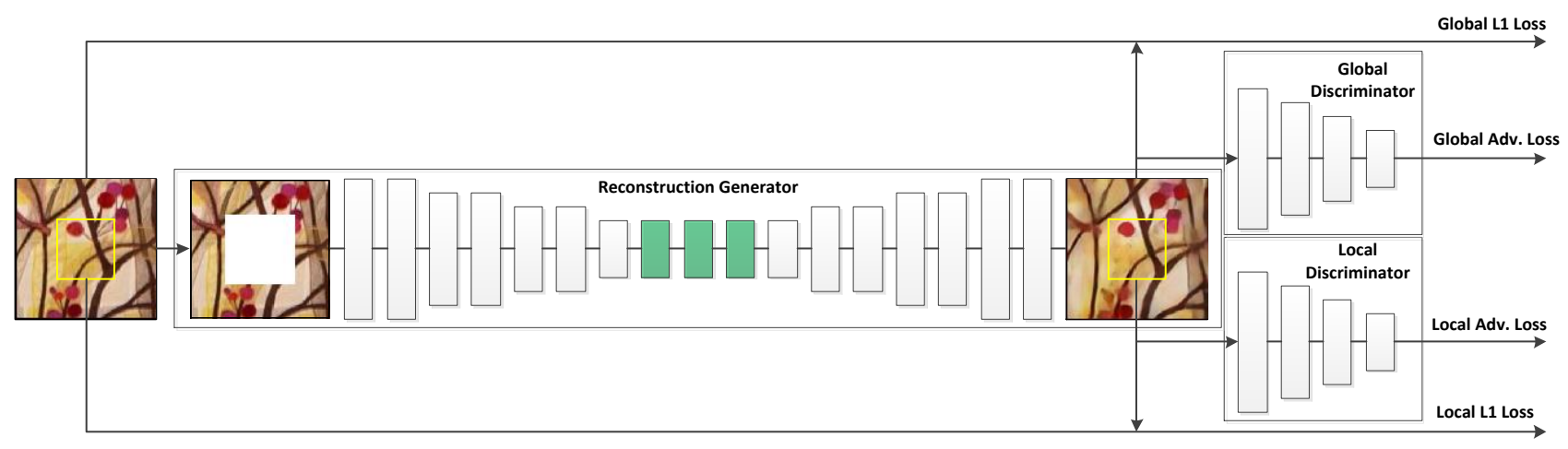

Figure 8. The proposed adversarial neural network for reconstruction damaged arias.

In our work, to construct a efficient method of reconstruction of damaged areas in the image, we rely on the latest achievements in the field of image reconstruction based on adversarial neural networks [27, 28]. Figure 8 shows the architecture of the proposed adversarial neural network for image reconstruction. The reconstruction generator model has 18 convolution layers with kernel size 3 for all layers: C64-C64-MP-C64-C128-MP- C128- C128-MP-C256-C256D4C256D8-C256D12-C256-US-C128-C128-US-C128-C64-US-C64-C64-C3 (C-feature map, MP-max pooling, D-dilation rate, US-up sampling). The global discriminator has 4 convolution layers and 1 fullyconnected layer: C64K5S2-MPC128K5S2-MP-C256K5S2-MP-C256K3-FC1 (K-kernel size, S-strides, FC-fullyconnected). The local discriminator also has 4 convolution layers and 1 fullyconnected layer: C64K5S2-MP-C128K5S2-MP-C256K3-MP-C256K3-FC1.

All neural networks (reconstruction generator, local and global discriminators) have the following identical parameters. The activation function is ELU [29], which is a more efficient modification of the activation function ReLU [30]. Additionally, when using ELU, there is no need to perform normalization batch. The ADAM method [31] with a training step of 0.0005 is used as an optimizer. As activation function in the last layers of neural networks, a logistic sigmoid is used.

To train the reconstruction generator, we use four types of losses: global absolute difference, local absolute difference, adversarial loss from the global discriminator, adversarial loss from the local discriminator. Generator losses are calculated according to the expression:

$$
\begin{aligned}
& \operatorname{Loss}_{G}=\lambda_{1} L_{l 1_{-} l o c}+\lambda_{2} L_{l 1_{-} g l o b}+\lambda_{3} L_{a d v_{-} l o c}+\lambda_{4} L_{a d v_{-} g l o b}, \\
& L_{l 1_{-} g l o b}=\left|x_{g l o b}-G\left(x_{d e f}\right)\right|, L_{l 1_{-} l o c}=\left|x_{l o c}-G\left(x_{d e f}\right)\right|, \\
& L_{a d v_{-} l o c}=\arg \max _{G} E \log \left(D_{l o c}\left(G\left(x_{d e f}\right)\right)\right), L_{a d v_{-} g l o b}=\arg \max _{G} E \log \left(D_{g l o b}\left(G\left(x_{d e f}\right)\right)\right)
\end{aligned}
$$

where $x_{g l o b}$ - undamaged source image, $\quad x_{l o c}$ - local undamaged area on the source image, $x_{d e f}$ - image with defect, $\lambda_{1}, \lambda_{2}, \lambda_{3}, \lambda_{4}$-coefficients of proportionality which are set manually. For both discriminators (local and global) losses are calculated according to the expression: 


$$
L_{D}=\arg \max _{D} E \log (D(x))+E \log \left(1-D\left(G\left(x_{d e f}\right)\right)\right)
$$

where $x$-source image, the size of which depends on what discriminator is used.

\section{EXPEREMENTS}

We used 75 images to demonstrate the effectiveness of the proposed method. These images contain data coming from organs with different reflective properties and varying illuminations: abdominal wall, ureter, kidney, and heart. We also received the ground truth for images from our dataset.

To evaluate the effectiveness of the proposed method we use the following metrics:

- The probability of correct detection:

$$
C P=\frac{T P}{D e f P x}
$$

- The probability of a false alarm:

$$
F A=\frac{F P}{A l l P i x-D e f P x}
$$

where $T P$ is true positive, FP is false positive, DefPix - total amountof pixels belonging to the reflection, AllPix total amount of pixels.

We compare the proposed specular reflection detection algorithm for test images and the well-known classical methods [6,9]. The experimental results (table 1) show that the probability of correct detection by the proposed method has the highest value. The new approach is better than the existing ones because it is an adaptive solution in complex cases such as change of appearance or texture.

Table 1. Comparison the probability of detection for different methods

\begin{tabular}{|c|c|c|}
\hline & The probability of false alarm, \% & The probability of correct detection, \% \\
\hline Zimmerman et al. [9] & 24 & 79 \\
\hline Arnold et al. [6] & 27 & 82 \\
\hline Proposed method & 22 & 86 \\
\hline
\end{tabular}

Image 9 shows some examples of reconstructed images using our network and method based on patch-matching [17]. For testing effectiveness our method image reconstruction we use DTD textures database (the images are cropped and have a size of $128 \times 128$ pixels, with a missing area of $64 \times 64$ pixels) [32].

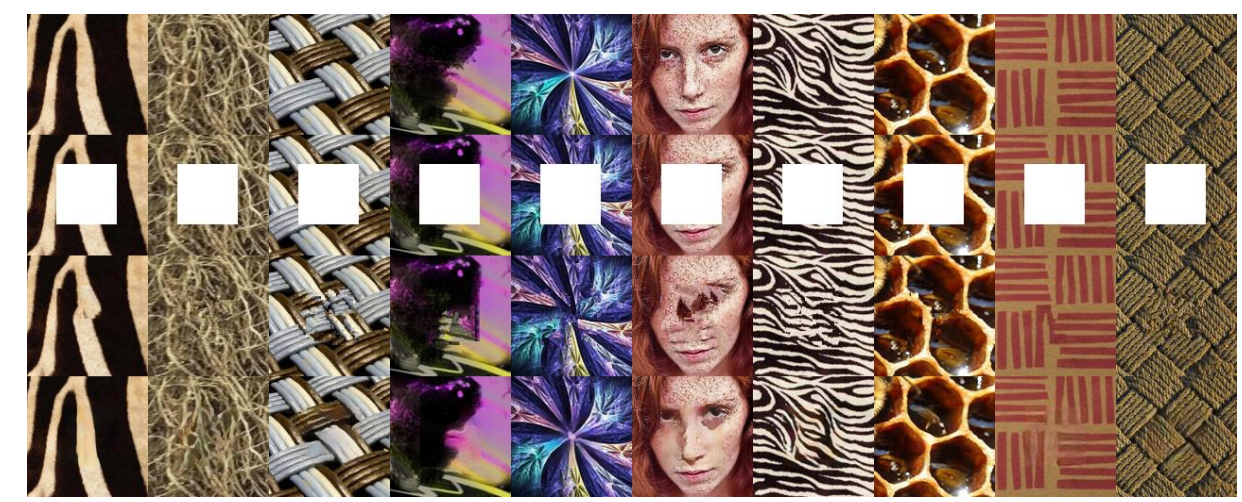

Figure 9. Examples of reconstructed images. The first line corresponds to the original images. The second line corresponds to the damaged image. The third line corresponds to method Criminisi et al.[17]. The lower line corresponds to the proposed adversarial neural network. 
Table 2 summarizes some quantitative metrics corresponding to image 9.

Table 2. Comparison quality of reconstruction of damaged images for different methods

\begin{tabular}{|c|c|c|c|c|c|c|c|c|c|c|}
\hline & \multicolumn{10}{|c|}{ Peak Signal-to-Noise Ratio (PSNR) } \\
\hline Criminisi et al. [\&] & 21.3253 & 19.5834 & 15.3500 & 19.0429 & 17.2933 & 17.4394 & 12.6819 & 18.4926 & 29.2273 & 20.3336 \\
\hline \multirow[t]{2}{*}{ Proposed Adv.Net. } & 33.0295 & 27.2005 & 27.6572 & 29.3446 & 26.3332 & 26.0032 & 25.6540 & 28.2538 & 33.3350 & 25.8708 \\
\hline & \multicolumn{10}{|c|}{ Mean-squared error (MSE) } \\
\hline Criminisi et al. [\&] & 0.0074 & 0.0110 & 0.0292 & 0.0125 & 0.0186 & 0.0180 & 0.0539 & 0.0141 & 0.0012 & 0.0093 \\
\hline \multirow[t]{2}{*}{ Proposed Adv.Net. } & 0.0005 & 0.0019 & 0.0017 & 0.0012 & 0.0023 & 0.0025 & 0.0027 & 0.0015 & 0.0004 & 0.0026 \\
\hline & \multicolumn{10}{|c|}{ Structural Similarity Index (SSIM) } \\
\hline Criminisi et al. [\&] & 0.9331 & 0.8350 & 0.7856 & 0.8270 & 0.8370 & 0.8582 & 0.7810 & 0.8988 & 0.9861 & 0.8356 \\
\hline Proposed Adv.Net. & 0.9679 & 0.9531 & 0.9448 & 0.8949 & 0.9642 & 0.9377 & 0.9770 & 0.9677 & 0.9917 & 0.9375 \\
\hline
\end{tabular}

Image 10 shows examples of detecting and reconstructing images containing specular reflections using the proposed method. Reconstruction of artifacts of irregular shape shown in image 10 was performed using square and rectangular masks exceeding the non-regular area. After reconstruction of large square/rectangular areas, the obtained values were transferred to damaged irregular masks.
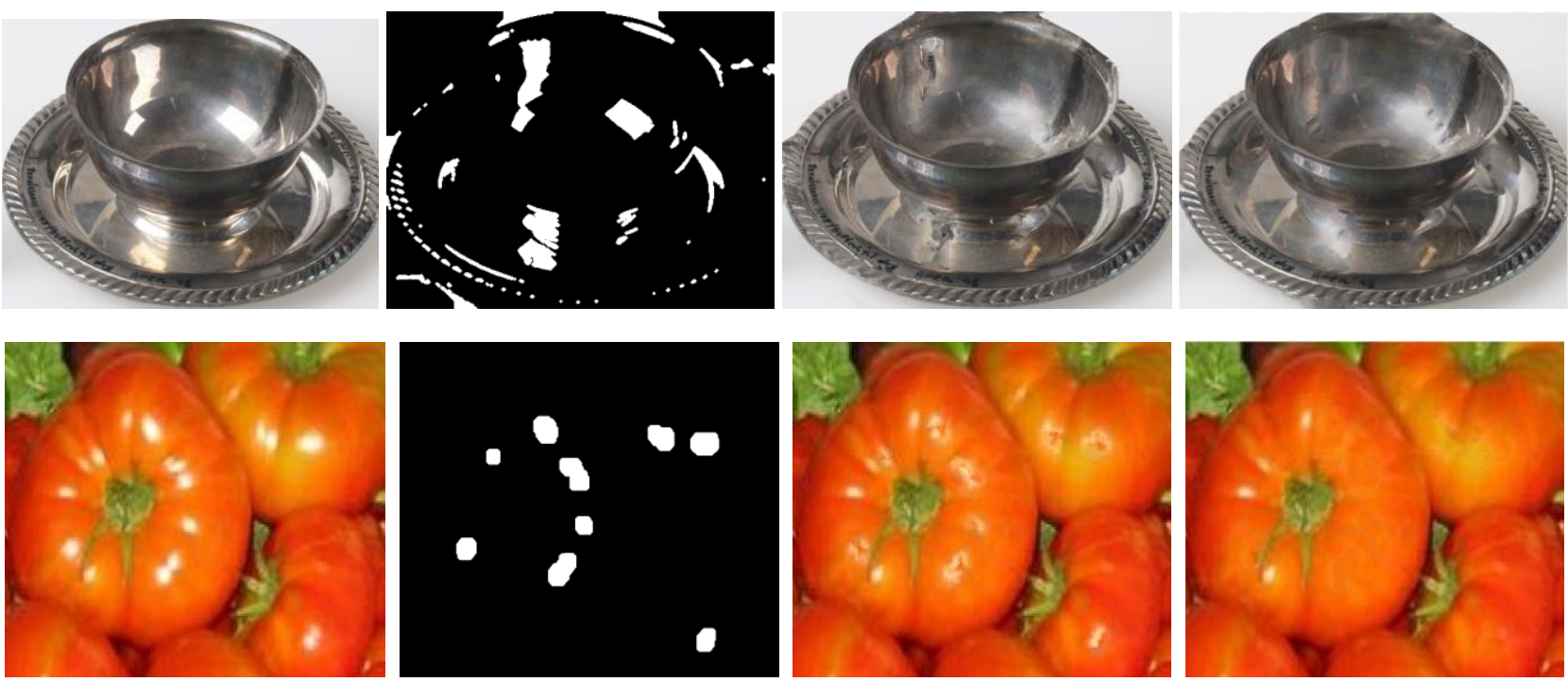

Figure 10. Examples of reconstructed images. The first column corresponds to the original images. The second column corresponds to detected specular reflection. The third column corresponds to method Criminisi et al. [17]. The last column corresponds to the proposed adversarial neural network.

The analysis of the obtained results confirms the high efficiency of the proposed method of detection and reconstruction of specular reflections.

\section{CONCLUSION}

We present a new automatic specular reflection detection algorithm for different images. The basic idea is to apply the modified alpha-rooting image enhancement approach and an efficient reflection detection algorithm in the HSV color space. The proposed image enhancement results compare favorably against other state-of-the-art approaches. The use of 
adversary neural networks allowed to achieve reconstruction of damaged areas with a high level of quality. In addition, it should be noted the high speed of the entire method, which can expand its application in practice.

\section{REFERENCES}

[1] Saint-Pierre, C.-A., Boisvert, J., Grimard, G., and Cheriet, F. Detection and correction of specular reflections for automatic surgical tool segmentation in thoracoscopic images. Machine Vision and Applications, 22(1):171-180. (2011)

[2] Karsch, K., Hedau, V., Forsyth, D., and Hoiem, D., Rendering synthetic objects into legacy photographs. ACM Transactions on Graphics (TOG), 30(6):157. (2011)

[3] Deng, Y., Manjunath, B., and Shin, H. (1999). Color image segmentation. In Computer Vision and Pattern Recognition, CVPR

[4] T.H. Stehle. Specular Reflection Removal in Endoscopic Images. Acta Polytechnica, vol. 46, pp. 32-36, (2006)

[5] A. Artusi, F. Banterle, D. Chetverikov. A Survey of Specularity Removal Methods. Computer graphics forum, vol. 30, number 8, pp. 2208-2230, (2011)

[6] M. Arnold, A. Ghosh, S. Ameling, and G. Lacey. Automatic Segmentation and Concealment of Specular Highlights for Endoscopic Imaging. EURASIP Journal on Image and Video Processing, pp. 1-12, (2010)

[7] G. Karapetyan, H. Sarukhanyan. Automatic Detection and Concealment of Specular Reflections for Endoscopic Images. In Computer Science and Information Technologies (CSIT), pp. 1-8, (2013)

[8] D. Stoyanov, G.Z. Yang, Removing specular reflection components for robotic assisted laparoscopic surgery, in: IEEE International Conference on Image Processing (ICIP), Vol. 3, IEEE, pp. III-632, ( 2005)

[9] G. Zimmerman-Moreno, H. Greenspan. Automatic detection of specular reflections in uterine cervix images, in Medical Imaging, 61446E-61446E, International Society for Optics and Photonics, (2006)

[10] J.M. Marcinczak, R.-R. Grigat. Closed contour specular reflection segmentation in laparoscopic images, Journal of Biomedical Imaging, vol. 18, (2013)

[11] A. Das, A. Kar, D. Bhattacharyya. Preprocessing for Automating Early Detection of Cervical Cancer. Information Visualisation (IV), 2011 15th International Conference on, (2011)

[12] R. Richa, P. Poignet, C. Liu, Three-dimensional motion tracking for beating heart surgery using a thin-plate spline deformable model, The International Journal of Robotics Research 29 (2-3) 218-230, (2010)

[13] S. Rohl, S. Bodenstedt, S. Suwelack, H. Kenngott, B. P. Muller-Stich, R. Dillmann, S. Speidel. Dense GPUenhanced surface reconstruction from stereo endoscopic images for intraoperative registration, Medical physics 39 (3) $1632-1645,(2012)$

[14] A. Mojtaba, M. Majid, S. Reza S.M., K. Nader, S. Shadrokh, N. Kayvan, Adaptive specular reflection detection and inpainting in colonoscopy video frames, CoRR, arXiv: 1802.08402, [Online]. Available: https://arxiv.org/abs/1802.08402, (2018)

[15]A. Morgand and M. Tamaazousti, Generic and real-time detection of specular reflections in images, IEEE, International Conference on Computer Vision Theory and Applications (VISAPP), (2014)

[16] M. Bertalmio, G. Sapiro, V. Caselles, and C. Balleste, Image inpainting, New Orleans: Proceedings of SIGGRAPH, 889p, (2000)

[17] A. Criminisi, P. Perez, K. Toyama, Region filling and object removal by exemplar-based image inpainting, IEEE Transactions on Image Processing, vol. 13, pp. 1200-1212, (2004)

[18] D. Pathak, P. Krahenbuhl, J. Donahue, T. Darrell. A. A. Efros, Context Encoders: Feature Learning by Inpainting, CoRR, arXiv: 1604.07379v2, [Online]. Available: https://arxiv.org/abs/1604.07379v2, (2016)

[19] A. Grigoryan and S. Agaian. Image enhancement. Advances in Imaging and Electron Physics. New York: Academic: pp. 165-243, (2004)

[20] J. Tang, E. Peli, and S. Acton. Image enhancement using a contrast measure in the compressed domain. IEEE Signal Process. Lett., vol. 10, no. 10: pp. 289-292, (2003)

[21] T. Zong, H. Lin, and T. Kao. Adaptive local contrast enhancement method for medical images displayed on a video monitor. Med. Eng. Phys., vol. 22: pp. 79-87, (2000) 
[22] W. M. Morrow, R. B. Paranjape, R. M. Rangayyan, and J. E. L. Desautels. Region-based contrast enhancement of mammograms. IEEE Trans. Med. Imag., vol. 11, no. 3: pp. 392-406, (1992)

[23] V. Voronin, E. Semenishchev, M. Ponomarenko, S. Agaian. Combined local and global image enhancement algorithm. Electronic Imaging, Image Processing: Algorithms and Systems XVI, pp. 1-5(5).

[24] V. Voronin, E. Semenishchev, S. Tokareva, A. Zelenskiy, S. Agaian. Underwater image enhancement algorithm based on logarithmic transform histogram matching with spatial equalization. Proceedings of the IEEE 14th international conference on signal processing, ISBN: 978-1-5386-4672-4, pp. 434-438, (2018)

[25] V. Voronin, E. Semenishchev, V. Frants and S. Agaian. Smart cloud system for forensic thermal image enhancement using local and global logarithmic transform histogram matching. Proceedings of the IEEE International Conference on Smart Cloud, DOI 10.1109/SmartCloud.2018.00033, pp. 153-157, (2018)

[26] S.S. Agaian, B. Silver, and K.A. Panetta. Transform Coefficient Histogram-Based Image Enhancement Algorithms Using Contrast Entropy. IEEE transactions on image processing, vol. 16, no. 3, (2007)

[27] S. Iizuka, E. Simo-Serra, and H. Ishikawa. Globally and locally consistent image completion. ACM Transactions on Graphics (TOG), 36(4):107, 2017.

[28] Y. Jiahui, L. Zhe, Y. Jimei, S. Xiaohui, L. Xin, S. H. Thomas, Generative image inpainting with contextual attention, CoRR, arXiv: 1801.07892, [Online]. Available: https:// arxiv.org/abs/1801.07892

[29] D.-A. Clevert, T. Unterthiner, and S. Hochreiter. Fast and accurate deep network learning by exponential linear units (elus). CoRR, arXiv: 1511.07289, [Online]. Available: https://arxiv.org/abs/1511.07289, (2015)

[30] A. Krizhevsky, I. Sutskever, and G. Hinton, "Imagenet classification with deep convolutional neural networks," Advances in Neural Information Processing Systems, 1097-1105, (2012).

[31] Kingma, D.P. and Ba, J., "Adam: A method for stochastic optimization," CoRR, arXiv:1412.6980. [Online]. Available: https://arxiv.org/abs/1412.6980, (2014).

[32] M. Cimpoi, S. Maji, I. Kokkinos, S. Mohamed, and A. Vedaldi, Describing textures in the wild. In Proceedings of the IEEE Conference on Computer Vision and Pattern Recognition, pages 3606-3613, (2014)

\section{AUTHORS’ BACKGROUND}

\begin{tabular}{|l|l|l|l|}
\hline Your Name & Title* & Research Field & Personal website \\
\hline & & & \\
\hline & & & \\
\hline & & & \\
\hline & & & \\
\hline
\end{tabular}

*This form helps us to understand your paper better, the form itself will not be published.

*Title can be chosen from: master student, Phd candidate, assistant professor, lecture, senior lecture, associate professor, full professor 